\title{
An Unusual Case of Necrotizing Pneumonia Presenting with Acute Kidney Injury
}

\author{
Ugur Balkanci ${ }^{1}$, David $\mathrm{Sas}^{2}$, and Nadir Demirel ${ }^{2}$ \\ ${ }^{1}$ University of Minnesota Twin Cities \\ ${ }^{2}$ Mayo Clinic Rochester
}

November 10, 2020

An Unusual Case of Necrotizing Pneumonia Presenting with Acute Kidney Injury

Ugur Berkay Balkanci, MD

School of Public Health, University of Minnesota, Minneapolis, MN

David J. Sas, DO

Division of Pediatric Nephrology and Hypertension, Department of Pediatrics and Adolescent Medicine, Mayo Clinic, Rochester, Minnesota

Nadir Demirel, MD

Division of Pediatric Pulmonology, Department of Pediatrics and Adolescent Medicine, Mayo Clinic, Rochester, Minnesota

Corresponding Author:

Nadir Demirel, MD

Division of Pediatric Pulmonology

200 First Street SW

Rochester, MN 55906

Tel. No.: 5075380754

Fax No.: 5072840727

Demirel.nadir@mayo.edu

Key words: postinfectious glomerulonephritis, pneumothorax, complications, complicated pneumonia

Financial Disclosure: The authors have indicated they have no financial relationships relevant to this article to disclose.

Funding: No external funding.

Short title: "An unusual case of necrotizing pneumonia"

To the Editor:

Lower respiratory tract infections are the most common reason for hospitalization in the pediatric age group in the United States. Although pneumonia is prevalent, complicated pneumonia such as empyema, 
lung abscess and necrotizing pneumonia (NP) is uncommon in children ${ }^{1}$. The prevalence of complicated pneumococcal pneumonia decreased significantly after the introduction of the thirteen-valent pneumococcal vaccine in $2010^{1}$. NP in the pediatric population is a severe disease characterized by extensive destruction and liquefaction of the lung tissue resulting in loss of the pulmonary parenchymal architecture, cavitation of the lung, and pleural involvement. Renal complications of complicated pneumonia are rare and mostly reported as atypical hemolytic uremic syndrome (HUS) ${ }^{2}$. Post-infectious glomerulonephritis (PIGN) is an unexpected complication of bacterial pneumonia ${ }^{3}$.

We report a six-year-old otherwise healthy fully vaccinated girl with a 4-day history of fever, abdominal pain, vomiting, non-bloody diarrhea, and poor oral intake. Parents reported decreased urine output and darkcolored urine on the day of admission. Initial evaluation revealed serum creatinine of $5.01 \mathrm{mg} / \mathrm{dL}$ and blood urea nitrogen of $86 \mathrm{mg} / \mathrm{dL}$, elevated acute phase reactants suggesting acute kidney injury (AKI) in the setting of an undiagnosed acute infectious process. The patient was admitted with decreased effective circulatory volume. Urinalysis revealed hematuria with $<25 \%$ dysmorphic red blood cells (RBCs), proteinuria, pyuria, and RBC casts and granular casts, suggestive of acute glomerulonephritis.

She was started on intermittent hemodialysis at day 2 of admission to address uremia, fluid overload, and hyperphosphatemia. A renal biopsy revealed diffuse exudative glomerulonephritis, consistent with infectionrelated glomerulonephritis. ASO, Anti-DNase B were negative; C3, C4 levels were low. She was treated with pulse IV methylprednisolone $10 \mathrm{mg} / \mathrm{kg} /$ day for three days. The first 5 days in the hospital, the patient remained afebrile and her lung exam was normal without respiratory symptoms.

On day six of admission, she developed acute right-sided chest pain and shortness of breath during hemodialysis. Chest x-ray (CXR) revealed a large right-sided tension pneumothorax, prompting therapeutic chest tube placement. Repeat CXR revealed reexpansion of the right lung and a significant right upper lobe consolidation with an ovoid hyperlucency and an air-fluid level. A chest CT scan confirmed the diagnosis of NP with multiple cavities (Image).

Flexible bronchoscopy was performed with bronchoalveolar lavage revealing $42 \%$ neutrophils and negative cultures. She was treated with broad spectrum intravenous antibiotics.

During admission, she developed hypertension, well-controlled with scheduled enalapril and amlodipine, as well as isradipine as needed. On day 14 of admission, hemodialysis was discontinued as kidney function improved, and chest tube was removed. She was discharged at day 26 of admission on intravenous ceftriaxone and oral metronidazole to complete 30 days of treatment. A repeat chest $\mathrm{CT}$ at end of treatment showed complete resolution of NP. Renal functions and blood pressure normalized on follow up.

$\mathrm{NP}$ is characterized by persistent high fevers and prolonged hospitalizations even with appropriate antibiotic treatment $^{1}$. Most often, NP affects immunocompetent children with no underlying risk factors ${ }^{4}$. The pathophysiology of this complication is acute liquefactive necrosis of the lung parenchyma which results in the development of pneumatoceles ${ }^{4}$. The most common pathogen causing NP is Streptococcus pneumoniae followed by Staphylococcus aureus and Streptococcus pyogenes. Other rarer bacterial and viral pathogens are Mycoplasma pneumonia, Influenza, and Adenovirus ${ }^{1}$. Identifying the microbiologic pathogen can be challenging and is only made in $50 \%$ of cases $^{1}$. In our case, we did not isolate the causative microorganism. NP typically resolves without residual morbidity, even after a protracted course ${ }^{1,4}$.

Pleural involvement is almost universal in NP, and the course of pleural disease often determines duration and outcome, particularly as it relates to the complication of bronchopleural fistula (BPF) ${ }^{1}$. BPF is most likely due to the necrotic development of a connection between bronchial space and pleural space ${ }^{4}$ BPF formation is associated with a significantly longer hospital stay in children with $\mathrm{NP}^{4}$. Yet, most cases heal without surgical intervention ${ }^{4}$. Tension pneumothorax has been observed as a rare complication of $\mathrm{NP}^{1}$.

Renal involvement in complicated pneumonia is rare. Atypical HUS has been reported as a complication of pneumonia, particularly associated with empyema. (most commonly due to invasive Streptococcus pneumoniae $)^{2}$. In a case series of 37 cases of atypical HUS, 34 patients $(92 \%)$ had pneumonia with 10 pa- 
tients $(29 \%)$ with $\mathrm{NP}^{5}$. Less commonly, pneumonia can be associated with PIGN. PIGN is the most common glomerulonephritis in children worldwide. Pneumonia-associated PIGN is rare. In a case series from the US, PIGN accounted for $0.15 \%$ of admissions for pneumonia and $0.39 \%$ of admissions for glomerulonephritis ${ }^{6}$. Pneumonia-associated PIGN is known to be caused by various bacterial pathogens including Streptococcus pneumoniae, Staphylococcus aureus, Mycoplasma pneumoniae, Chlamydia pneumoniae, Nocardia, and Coxiella burnetii ${ }^{3}$. Different from the usual presentation of the PIGN (in which the time interval between a pharyngeal group A Streptococcal infection and PIGN is 6 to 10 days), pneumonia-associated PIGN is usually concomitant with the pulmonary disease ${ }^{3,6}$.

Our case is unusual in several ways: pneumonia-associated PIGN typically presents with respiratory symptoms first, and acute kidney injury developing during the course of pneumonia ${ }^{3}$. More surprisingly, the patient developed NP which is characterized by even more severe respiratory symptoms ${ }^{1}$. Yet, our patient presented without respiratory complaints and pneumonia became apparent only after the development of pneumothorax. We could only identify 2 cases of pneumonia-associated PIGN who presented with renal involvement before pulmonary complaints ${ }^{6,7}$. Also, previous cases in the literature of pneumonia-associated PIGN report mostly a non-complicated course of pulmonary disease ${ }^{3,6}$. In a case series of 11 children with pneumonia-associated PIGN, only one case developed a small empyema ${ }^{6}$. Similarly, the majority of the reported cases of pneumonia-associated PIGN describe a benign course of renal disease ${ }^{3,6}$. Our patient's kidney failure progressed rapidly, and she required 2 weeks of intermittent hemodialysis and a three-day course of pulse steroid therapy. At present, systemic corticosteroids are not recommended for patients with complicated pneumonia. A Cochrane review including 17 randomized controlled trials, of which four were conducted on children, found that corticosteroid therapy reduced mortality and morbidity in adults with severe CAP, and morbidity, but not mortality, in adults and children with non-severe CAP ${ }^{1}$. We speculate that pulse steroid treatment may have modified the course of NP in our patient.

This case suggests an atypical presentation of NP with predominant renal complications is possible. Pediatricians should be aware of renal complications of respiratory diseases. Systemic steroids should be considered in the treatment of NP.

References:

1. de Benedictis FM, Kerem E, Chang AB, Colin AA, Zar HJ, Bush A. Complicated pneumonia in children. Lancet 2020;396:786-798.

2. Spinale JM, Ruebner RL, Kaplan BS, Copelovitch L. Update on Streptococcus pneumoniae associated hemolytic uremic syndrome. Curr Opin Pediatr 2013;25:203-208.

3. Carceller Lechón F, de la Torre Espí M, Porto Abal R, Écija Peiró JL. Acute glomerulonephritis associated with pneumonia: a review of three cases. Pediatr Nephrol 2010;25:161-164.

4. Sawicki GS, Lu FL, Valim C, Cleveland RH, Colin AA. Necrotising pneumonia is an increasingly detected complication of pneumonia in children. Eur Respir J 2008;31:1285-1291.

5. Banerjee R, Hersh AL, Newland J, Beekmann SE, Polgreen PM, Bender J, Shaw J, Copelovitch L, Kaplan BS, Shah SS. Streptococcus pneumoniae-associated Hemolytic Uremic Syndrome Among Children in North America. Pediatr Infect Dis J 2011;30:736-739.

6. Srivastava T, Warady BA, Alon US. Pneumonia-associated acute glomerulonephritis. Clin Nephrol 2002; $57: 175-182$.

7. Schachter J, Pomeranz A, Berger I, Wolach B. Acute glomerulonephritis secondary to lobar pneumonia. Int J Pediatr Nephrol 1987;8:211-214. 


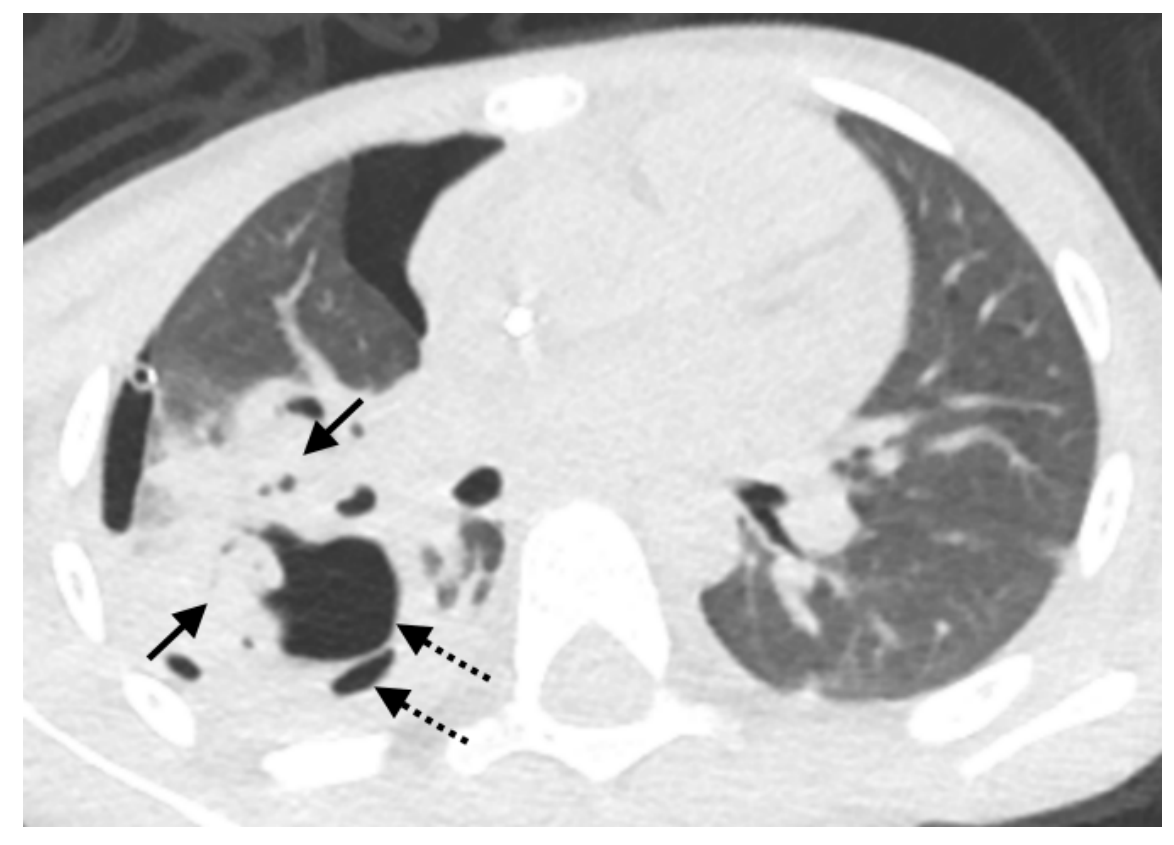

